\title{
Blood biomarker measurement as disease target: the case for generic treatment of high natriuretic peptide levels without a diagnosis of heart failure
}

\author{
AL Clark \\ Chair of Clinical Cardiology and Honorary Consultant Cardiologist, Department of Cardiology, Hull York Medical School, University of Hull, \\ Castle Hill Hospital, UK
}

KEYWORDS: Heart failure, natriuretic peptides, HeFNEF

DECLARATION OF INTERESTS No conflicts of interest declared.

\author{
Correspondence to AL Clark \\ Department of Cardiology, \\ Hull York Medical School, \\ University of Hull, \\ Castle Hill Hospital, \\ Cottingham, HUI6 5JQ, UK
}

tel. +44 (0) I 482622044

e-mail

a.l.clark@hull.ac.uk
Natriuretic peptides (NP) are hormones released by the heart. Atrial, or A-type, NP (ANP) is secreted predominantly by the atria in response to stretch and B-type NP (BNP) is released mainly from the left ventricle in response to an increase in left ventricular wall stress. The physiological effect of the NPs is natriuresis, but they have additional vasodilatory properties and reduce activation of the sympathetic and renin-angiotensin-aldosterone system.

\section{DIAGNOSTIC ROLE OF NATRIURETIC PEPTIDES}

Interest in NPs has mainly focused on their role in patients presenting with breathlessness in whom there is a potential diagnosis of heart failure. There is a strong relation between NP levels and a diagnosis of heart failure, with BNP and its metabolic cognates performing better than ANP at predicting its presence. The most convincing data relate to patients with left ventricular systolic dysfunction (LVSD) and raised NP levels (levels are also raised in patients with asymptomatic LVSD). The presently accepted value of NP measurement is therefore as a rule-out test: a breathless patient with a normal NP level is highly unlikely to have heart failure. Further studies have looked at the possible role for NP testing as a screening test for LVSD in general populations.

A key question in such research is the nature of the "gold standard' diagnostic procedure against which the NP measurement is being assessed. Typically, it is an echocardiographic measurement of left ventricular systolic function, such as left ventricular ejection fraction (particularly for population studies), or a panel diagnosis using all available data (particularly in diagnostic case studies). The unstated presumption is always that the 'gold standard' is the best available: but what if the NP level is the gold standard and not the echocardiogram?

\section{HEART FAILURE WITH A NORMAL EJECTION FRACTION}

One of the puzzles of heart failure research has been the phenomenon of heart failure with a normal ejection fraction (HeFNEF), variously described as 'diastolic heart failure' and 'heart failure with preserved ejection fraction'. Although HeFNEF is believed to be the cause in around half of patients with heart failure, not only has it been difficult to recruit patients to clinical trials of HeFNEF, but none of the treatments shown to be effective in managing heart failure with a reduced ejection fraction have been shown to work in patients with HeFNEF.

Perhaps the problem is that many of the patients recruited into HeFNEF trials simply did not have heart failure. Breathlessness is very common in older people,' and it is easy to label someone with breathlessness as having HeFNEF if the echocardiogram shows a normal left ventricular ejection fraction and, for example, left ventricular hypertrophy. 'Abnormalities' of diastolic function are almost universal in older people, making the diagnosis of HeFNEF a foregone conclusion in a breathless older person.

What is needed is some independent indicator that a problem in the heart is the cause of the symptoms, or at least, that the symptoms may be associated with an adverse prognosis. The NPs are the strongest single marker of prognosis in heart failure, but they are also related to outcome in other conditions such as acute coronary syndromes and pulmonary embolism in the general population. ${ }^{2}$ There is a strong inverse relation between NP level and measures of LV systolic function. Measurement of NP levels allows a distinction between a person with a left ventricular ejection fraction of $40 \%$ which is normal for that individual (normal NP, good 
prognosis) or abnormal (raised NP, adverse prognosis). Perhaps the reason that the trials of patients with HeFNEF have been neutral is that many participants did not have prognostically important disease. In the perindopril in elderly people with chronic heart failure (PEP-CHF) study for example, at least half the patients had an $\mathrm{N}$-terminal pro-brain natriuretic peptide (NtproBNP) level below the cut-off required to diagnose heart failure at the time, and consequently had a good prognosis. ${ }^{3}$ It was not surprising that perindopril had little effect on outcomes in such a population.

\section{SHOULD WE TREAT HYPER BNP-ANAEMIA?}

Should we stop considering details of cardiac structure and pathology and simply use the NP level as a diagnostic tool? A raised NP level (regardless of the patient's symptoms) is associated with an adverse prognosis and is thus possibly a target for treatment. In trials of patients with heart failure, treating to a target NP level is associated with a better prognosis than standard care. ${ }^{4.5} \mathrm{~A}$ raised NP level is on its own potentially an indication for treatment with medication to reduce it, such as angiotensin converting enzyme (ACE) inhibitors and beta-adrenoceptor antagonists.

There are, however, serious problems with such an approach. Heart failure is not the only condition to cause a raised NP level. Renal impairment in particular, but also sepsis, and even cancer ${ }^{6}$ are associated with a raised NP. While it is likely that the NP is released by the heart, it doesn't mean that treating the NP per se will be associated with improvement in prognosis. The correct diagnosis could be missed by not investigating the patient with a raised NP fully, with resulting potential harm; and treatment with an ACE inhibitor might worsen the patient's problem if the cause of the raised NP is, for example, renal failure, pulmonary embolus or hypertension, or aortic stenosis.

\section{CONCLUSION}

The role of NPs will develop in the next few years. Testing has an established role in ruling-out the diagnosis in patients presenting with symptoms suggestive of heart failure, but it is also going to be used to sort out the problem of HeFNEF. The true prevalence of HeFNEF may be established. Trials will have to be repeated in patients with HeFNEF with a raised NP being used as an admission criterion; so far trials are likely to have included large numbers of patients for whom treatment was unlikely to help.

Screening populations is, however, likely to be the biggest new use for NP testing. The prevalence of cardiovascular disease rises with age, and populations are accustomed to screening with blood tests (and subsequent treatment), particularly for cholesterol. Measuring NP levels could become part of the framework for routine screening at age 50 . However, because treating with ACE inhibitors may be harmful, a raised NP should trigger further investigation, not immediate treatment. Even with the new approach however, there will still be a population with no symptoms, in sinus rhythm, a normal chest radiograph, electrocardiogram (ECG) and echocardiogram and normal renal function, but a raised NP level. No one knows the size of this group, whether such patients should be treated, or with what. The only way to answer the questions is further trials.

\section{REFERENCES}

I Ho SF, O'Mahony MS, Steward JA et al. Dyspnoea and quality of life in older people at home. Age Ageing 200I; 30:155-9. http:// dx.doi.org/10.1093/ageing/30.2.155

2 McDonagh TA, Cunningham AD, Morrison CE et al. Left ventricular dysfunction, natriuretic peptides, and mortality in an urban population. Heart 200I; 86:2I-6. http://dx.doi.org/I0.II36/heart.86.I.2I

3 Cleland JG, Taylor J, Tendera M. Prognosis in heart failure with a normal ejection fraction. N Engl J Med 2007; 357:829-30. http:// dx.doi.org/l0.1056/NEJMc076/79

4 Porapakkham P, Porapakkham P, Zimmet H et al. B-type natriuretic peptide-guided heart failure therapy: a meta-analysis. Arch Intern Med 2010; 170:507-14. http://dx.doi.org/I0.1001/archinternmed.2010.35

5 Januzzi JL Jr, Rehman SU, Mohammed AA et al. Use of aminoterminal pro-B-type natriuretic peptide to guide outpatient therapy of patients with chronic left ventricular systolic dysfunction. J Am Coll Cardiol 2011; 58:188I-9. http://dx.doi. org/10.1016/j.jacc.2011.03.072

6 Burjonroppa SC, Tong AT, Xiao LC et al. Cancer patients with markedly elevated B-type natriuretic peptide may not have volume overload. Am J Clin Oncol 2007; 30:287-93. http://dx.doi. org/I0.1097/0I.coc.0000256I0I.04404.b0 